\title{
Alexander Davidovich loffe: a Life of Contributions
}

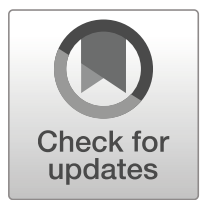

Huynh Van Ngai ${ }^{1} \cdot$ Hoang Xuan $\mathrm{Phu}^{2} \cdot$ R. Tyrrell Rockafellar ${ }^{3} \cdot$ Michel Théra $^{4,5}$ (D)

Received: 1 April 2019 / Accepted: 2 April 2019 / Published online: 13 May 2019

(C) Vietnam Academy of Science and Technology (VAST) and Springer Nature Singapore Pte Ltd. 2019

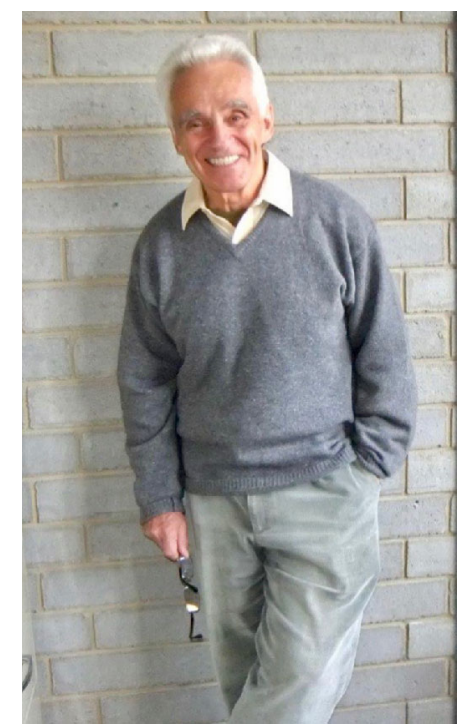

Michel Théra

michel.thera@unilim.fr

Huynh Van Ngai

ngaivn@yahoo.com

Hoang Xuan Phu

hxphu@math.ac.vn

R. Tyrrell Rockafellar

rtr@uw.edu

Department of Mathematics, Quy Nhon University, Quy Nhon, Binh Dinh, Vietnam

2 Institute of Mathematics, Vietnam Academy of Science and Technology, Hanoi, Vietnam

3 Department of Mathematics, University of Washington, Seattle, WA 98195-4350, USA

4 Université de Limoges, Limoges, France

5 Centre for Informatics and Applied Optimisation, Federation University Australia, Ballarat, Australia 
Through this special issue, Vietnam Journal of Mathematics is paying tribute to Professor Alexander Davidovich Ioffe on the occasion of his 80th birthday. We would like to take this opportunity to give some hints on his life and on his scientific contributions that have a far-reaching influence on the theory of variational analysis and related areas.

Life Alex Ioffe was born in 1938 in Leningrad. In 1961, he graduated from Moscow Institute of Aviational Engineering with MA in electrical engineering (specifically, flight control) and started to work at a military research institute. As with many such institutes in the former Soviet Union, it did not have an official name, only a number. In 1963, he started to attend a special program for engineers at the Mathematical Department of Moscow State University. It was a very serious four-year program ( $4 \mathrm{~h}$ every evening, 4 days in a week), and very soon he realized that mathematics interested him much more than engineering. He finished this program late in 1966 and in 1967 got his $\mathrm{PhD}$ already in mathematics (although his dissertation was devoted to variational problems of flight control). In 1972, Leonid Vitalievich Kantorovich, a celebrated mathematician and a future Nobel Prize winner (in economics) moved to Moscow and invited Alex to his laboratory. Alex happily accepted this offer. But soon he was compelled to leave the laboratory because the security department required that he should renew his security clearance (which formally prohibited him from communicating with his colleagues abroad and imposed lots of other restrictions) and started to work as an associate professor of applied mathematics at Moscow Institute of Automobile and Road Construction.

In 1976, Alex and his wife Rosa applied for emigration to Israel and were refused by the authorities with a reference to his former job in a military institute. Typically, a person who applied to emigrate from the USSR was immediately fired from the job. This was the case with Rosa, also a teacher in an engineering college. But Alex was luckier: he was dismissed from his position and moved to some refresher courses for engineers in the same institute. Apparently, the Rector of the Institute managed to resist the KGB pressure to fire him completely. At the same time, in common with all other refusenik ${ }^{1}$ scientists, over a period of almost 12 years, Alex was unable to publish in Soviet journals despite protest actions by his colleagues outside of the USSR and also to attend conferences and seminars, except some unauthorized meetings organized by refusenik themselves, including two international Conferences on Collective Phenomena in 1980 and $1981 .^{2}$ These two meetings were attended by a number of outstanding researchers from the West. But the subsequent two meetings planned for 1983 and 1985 did not happen because the Soviet authorities did not give visas to anyone who could be suspected to be a participant. Needless to say that there were numerous protests and support actions in the World scientific community. In particular, proceedings of all four conferences were published in Annals of the New York Academy of Sciences with a foreword by Joel L. Lebowitz, that time the president of the Academy. An international conference on convex analysis and optimization was organized in 1980 in

\footnotetext{
${ }^{1}$ According to Joel L. Lebowitz, Professor of Mathematics and Physics at Rutgers, a refusenik is a person who has applied to the authorities for the permission to emigrate from the USSR, but who has been refused this permission. Joel used his authority as a member of the Bureau, then as a vice-president and president of the New York Academy of Sciences in contacting the president of the USSR Academy of Sciences, Professor A. Alexandrov on several occasions to help refusniks and did every effort to keep links with the scientists cut off by the Iron Curtain.

${ }^{2}$ The host of the apartment where the meetings took place, Dr. V. Brailovski was arrested in November 1980 and spent 5 years in exile.
} 
London by J.-P. Aubin and R. Vinter. ${ }^{3}$ Note also that Alex was refusenik in absentia from CEREMADE (Centre de Mathématiques de la Décision) at the University Paris Dauphine over a period of more than 10 years. In his recent book, Alex expresses his gratitude to a number of friends and colleagues inside and outside of the Soviet Union whose support and encouragement helped him to survive as a mathematician.

In 1986, his son Dima, already a family man, applied for emigration to Israel and was also refused on the ground that he did not have relatives in Israel. After that Alex started a hunger strike (in fact he undertook two hunger strikes). The hunger strike attracted great attention from both mathematical circles and Jewish organizations, mainly in the USA, France, UK, and Canada, and eventually his son and his family got permission to leave in January 1987. Alex, Rosa, and their daughter Anna were able to leave a year later and arrived in Israel on January 24, 1988. The Technion offered him a position that began in 1979 in which he remained for almost 9 years. Since 1988 and until 2006, Alex was Professor in the Department of Mathematics at the Technion. In 1994, he got a Technion management chair in mathematics. He has been Professor Emeritus since his retirement in 2006. In 2006, Alex was awarded a Honoris Causa Doctorate from Keio University in Tokyo.

Research The main interests and contributions of Alex Ioffe are in the areas of calculus of variations and optimal control; convex analysis; nonsmooth analysis (subdifferential calculus); nonlinear analysis (metric critical point theory); variational analysis (metric regularity). These topics are the main subjects of the two books he wrote, the widely read and influential "Theory of Extremal Problems", with Vladimir Mikhailovich Tikhomirov, published in 1974 in Russian and translated into English, German, and Japanese, and "Variational Analysis of Regular Mappings, Theory and Applications" published in 2017.

Calculus of Variations and Optimal Control Alex was probably the first to attract attention to the role of convex duality in relaxation theory (already in his first 1966 paper and in a more general situation in two 1968 papers with Tikhomirov). This approach was later systematically developed by Ekeland and Temam in their 1972 book.

In his paper "On lower semicontinuity of integral functionals", SIAM J. Control \& Optimization 15, 521-538 (1977), Alex gave a verifiable necessary and sufficient condition for lower semicontinuity of integral functionals. (G. Buttazzo in his 1989 book explained that a non-published earlier version of this result, for nonnegative integrands, was offered by $\mathrm{E}$. De Giorgi in his course at the Istituto Nationale di Alta Matematica in 1968-1969).

The 1974 book by Ioffe and Tikhomirov contained a (totally new) proof of the Pontryagin maximum principle in optimal control that systematically used the Lyusternik theorem. This seems to have been the first indication of the role of (what was later called) metric regularity to optimization. Following publication of the three part 1979 paper in SIAM J. Control \& Optimization 17, 245-288 (1977), this idea becomes a focal point in Alex's works connected with necessary conditions in optimization. The power of the approach based on this idea was powerfully demonstrated in the first proof of the maximum principle for nonconvex-valued differential inclusions given in "Euler-Lagrange and Hamiltonian formalisms in dynamic optimization", Trans. Amer. Math. Soc. 349, 2871-2900 (1997).

\footnotetext{
${ }^{3}$ Convex analysis and optimization (London, 1980), Res. Notes in Math. 57, Pitman, Boston, Mass.-London,
} 1982. 
To explain the problem, we note that starting with Clarke's 1976 papers, there was a considerable interest in optimal control problems for systems governed by differentiable inclusions. The difficulty was that the existing approaches permitted the application of the maximum principle to such problems only under the assumption that the set-valued mapping is convex-valued. The breakthrough came only 20 years later with the mentioned Alex's 1997 paper and a paper by Vinter and Zheng who proved the result in a different way under slightly stronger assumptions.

Another idea associated with Alex's recent publications (although its origins also go back to the 1974 book) is that in the framework of variational analysis, constrained problems can be equivalently reduced to simply formulated unconstrained problems, so that, from this perspective, the optimization problems arising in variational analysis are essentially "constraint-free". The seeds of this idea were already evident in Alex's earlier mentioned 1997 paper, in which the original optimal control problem was first reduced to an unconstrained variational problem optimal control problem of Bolza type with subsequent analysis based on an earlier result of Alex with R. Tyrrell Rockafellar on such problems in "The Euler and Weierstrass conditions for nonsmooth variational problems", Calculus of Variations and PDEs 4, 59-87 (1996).

Exploiting this idea Alex recently improved Clarke's 2005 "stratified necessary condition" that remained for more than a decade the most advanced result in the theory of necessary conditions for optimal control problems with differential inclusions and, moreover, extended it to problems with state constraints. (The corresponding paper is to appear in Journal of Optimization Theory Appl. later this year.)

Variational Analysis: Metric Regularity Theory Alex's work in the theory of metric regularity more or less continued throughout all of his professional life and his results played a key role in developments of the theory. It was proved in the 1974 book with Tikhomirov that under the assumptions of the Lyusternik theorem a distance estimate (coined "metric regularity" by Borwein and Zhang in 1987) is valid. Two years later, S. Robinson proved that a similar estimate holds for a class of set-valued mappings. These papers opened up the new theory.

In another paper "Regular points of Lipschitz functions", Trans. Amer. Math. Soc. 251, 61-69 (1979), Alex introduced a criterion for metric regularity for Lipschitz functions in terms of Clarke's generalized gradients. The important point was that this paper contained also an estimate for the regularity constant in terms of the distance of generalized gradients from zero. Further developments of the subdifferential regularity theory more or less followed this pattern but using, subsequently, more sophisticated subdifferentials.

Metric regularity theory in metric spaces (initiated by E. Levitin, A. Milyutin and N. Osmolovskii in mid-70s) was fundamentally developed in "Metric regularity and subdifferential calculus", Uspehi Mat. Nauk 55, 103-162 (in Russian), English translation: Russian Math. Surveys 55:3 (2000). Metric criteria developed in the paper often, though not always, turn out to be more precise and easier to use than rival subdifferential criteria even in Banach spaces. The current state of the theory and its applications to optimization and analysis are presented in his 2017 book.

Among the most recent contributions, we should mention the joint paper with M. Fabian and J. Revalski in Proc. Amer. Math. Soc. 146, 3157-3167 (2018), with the main result stating that all local characteristics of metric regularity of set-valued mappings between Banach 
spaces are defined by restrictions of the graph of the mapping to products of separable subspaces of the domain and range spaces.

Convex Analysis This work was done mainly in 1966-1972 and is closely connected with and aimed at applications to optimal control, with the main interest in developing the theory of convex integral functionals. The most important were probably the results about subdifferentials of such functionals obtained jointly with Tikhomirov in Funct. Anal. Appl. 3, 218-227 (1969), and Levin in "Subdifferentials of convex functions", Trans. Moscow Math. Soc. 26, 1-72 (1972).

Subdifferential Calculus for Non-Convex Functions Alex's main contribution is the introduction of the G-subdifferential which is the only subdifferential with rich calculus that makes sense in general Banach spaces. Moreover, in a weakly compactly generated space, separable space in particular, any other "good" subdifferential (e.g., the limiting Fréchet subdifferential if the space is Asplund or the limiting viscosity subdifferential if there is an equivalent norm in the space differentiable away from the origin in one or another sense) coincides with the G-subdifferential. On the other hand, in a "bad" space like $L^{\infty}$, no other subdifferential with a comparable collection of properties is known.

Metric Critical Point Theory This is the subject matter of a series of publications from the mid-90s (jointly with Schwartzmann) centered around problems associated with existence and stability of critical points of continuous functions on metric spaces.

One of the results we can mention is an extension of the Morse deformation lemma to a certain class of $C^{1,1}$ functions on Hilbert spaces (namely $C^{1,1}$ perturbation of quadratic functions): there is a Lipschitz homeomorphism that transforms such a function into a sum of squares if its critical points are nondegenerate (in some sense): "Parametric Morse lemmas for $C^{1,1}$-functions", Contemporary Mathematics 204, 139-147 (1997).

Finally, we would like to mention a series of papers, partly jointly with D. Drusviatskiy and A. Lewis on variational analysis of semi-algebraic and tame mappings, as well as the joint paper with Dmitri Drusviatskiy and Adrian Lewis "Transversality and alternating projections", Foundations Comp. Math. 15, 1637-1651 (2015), which makes plain the close connection between linear convergence and weak transversality properties of certain sets involved.

Anecdotes To end up this presentation, we would like to present two funny stories about Alex, provided to us by Alex himself.

- The first one concerns his first publication while he was still a student in a special course for engineers at the Mathematics Department of Moscow State University. In this contribution, entitled "Transformation of correctly posed variational problems", Soviet Math. Doklady 7, 623-627 (1966), he invented some techniques to work with convex functions in Banach spaces. According to Alex, Vladimir Tikhomirov (as already said, Tikhomirov was a close colleague of Alex with whom he already started to work), was very impressed by the results and suggested that Alex should present them at the forthcoming World Congress of Mathematicians to be held in Moscow in Summer of 1966. His 15-min contributed talk was accepted and took place in a big hall with several hundred people. The session was chaired by Len Berkowitz who later, when Alex was already a refusenik, became a friend. After the talk, when questions time started, Vadim Arkin stood up and said that his result resembled to a recent result by Jack Warga and 
asked whether he knew Warga's work. Alex left the podium totally humiliated since he was not able to explain the connection. He went afterwards through the literature and found Warga's relaxation results as well as the earlier results of Young and McShane. However, Alex's proof was original and much simpler, as it used convex duality. This is the story of the first lecture given by Alex.

- In October 1966, Boris Polyak invited Alex to give a talk at his university. After the talk, about convex duality, Boris (who was the secretary of the Control and Optimization section at the congress) informed him that during the congress has spoken with R. Tyrrell Rockafellar, who had told him about something that sounded similar. Vladimir Tikhomirov and Alex rushed immediately afterward into the library, found Rockafellar's papers and discovered that they contained all that was being talked about at the seminar and much more, and the results were presented in a much more elegant way. That was the beginning of his mathematical and very fruitful career. Fortunately, it was also the beginning of friendly relations with both Jack Warga and R. Tyrrell Rockafellar who played an important part in Alex's scientific life.

February 2019

Publisher's Note Springer Nature remains neutral with regard to jurisdictional claims in published maps and institutional affiliations. 